University of South Carolina

Scholar Commons

$12-3-2007$

\title{
). Size Dependency of the Elastic Modulus of ZnO Nanowires: Surface Stress Effect
}

\author{
Guofeng Wang
}

Xiaodong Li

University of South Carolina - Columbia, lixiao@cec.sc.edu

Follow this and additional works at: https://scholarcommons.sc.edu/emec_facpub

Part of the Applied Mechanics Commons, and the Engineering Physics Commons

\section{Publication Info}

Published in Applied Physics Letters, Volume 91, Issue 23, 2007, pages \#231912-.

(C)Applied Physics Letters 2007, American Institute of Physics.

Wang, G., \& Li, X. (3 December 2007). Size Dependency of the Elastic Modulus of ZnO Nanowires: Surface Stress Effect. Applied Physics Letters, 91 (23), \#231912. http://dx.doi.org/10.1063/1.2821118

This Article is brought to you by the Mechanical Engineering, Department of at Scholar Commons. It has been accepted for inclusion in Faculty Publications by an authorized administrator of Scholar Commons. For more information, please contact digres@mailbox.sc.edu. 


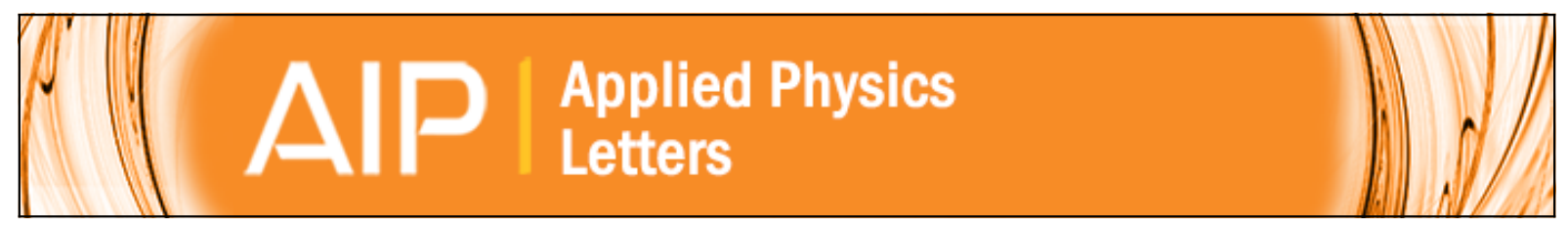

Size dependency of the elastic modulus of $\mathrm{ZnO}$ nanowires: Surface stress effect

Guofeng Wang and Xiaodong Li

Citation: Applied Physics Letters 91, 231912 (2007); doi: 10.1063/1.2821118

View online: http://dx.doi.org/10.1063/1.2821118

View Table of Contents: http://scitation.aip.org/content/aip/journal/apl/91/23?ver=pdfcov

Published by the AIP Publishing

\section{Articles you may be interested in}

Third-order elastic constants of $\mathrm{ZnO}$ and size effect in $\mathrm{ZnO}$ nanowires

J. Appl. Phys. 115, 213516 (2014); 10.1063/1.4881775

Surface effect on the size- and orientation-dependent elastic properties of single-crystal ZnO nanostructures J. Appl. Phys. 105, 034302 (2009); 10.1063/1.3077260

Finite size effect in $\mathrm{ZnO}$ nanowires

Appl. Phys. Lett. 90, 113101 (2007); 10.1063/1.2712507

Effects of bias stress on $\mathrm{ZnO}$ nanowire field-effect transistors fabricated with organic gate nanodielectrics Appl. Phys. Lett. 89, 193506 (2006); 10.1063/1.2378445

Intrinsic current-voltage properties of nanowires with four-probe scanning tunneling microscopy: A conductance transition of $\mathrm{ZnO}$ nanowire

Appl. Phys. Lett. 89, 043103 (2006); 10.1063/1.2234293

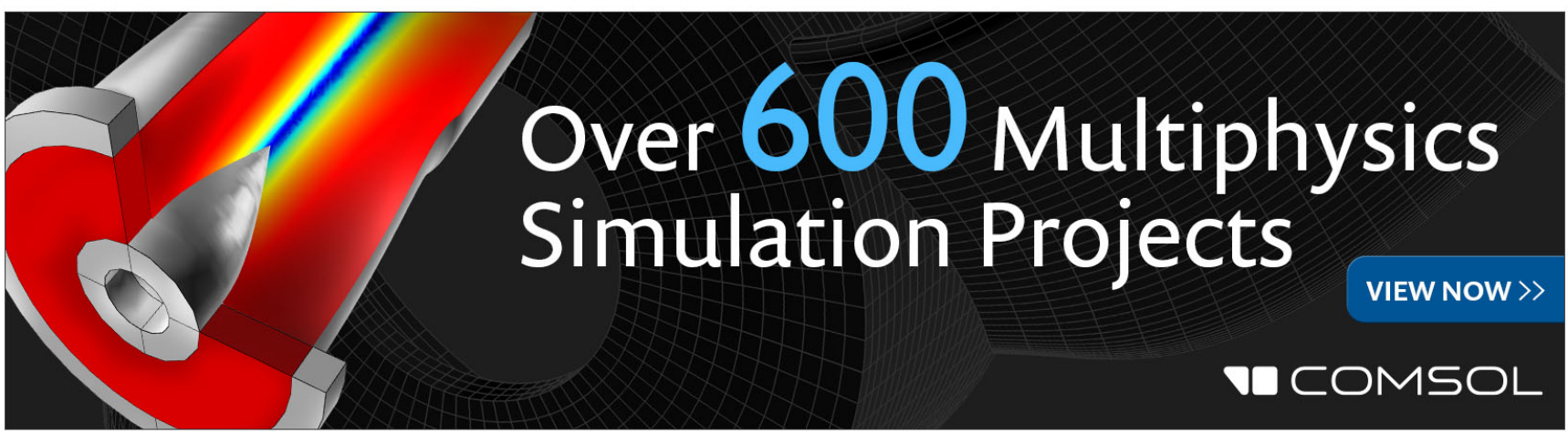




\title{
Size dependency of the elastic modulus of $\mathrm{ZnO}$ nanowires: Surface stress effect
}

\author{
Guofeng Wang $^{\text {a) }}$ \\ Department of Chemistry and Physics, University of South Carolina, Aiken, South Corolina 29801, USA \\ Xiaodong Li \\ Department of Mechanical Engineering, University of South Carolina, Columbia, \\ South Corolina 29208, USA
}

(Received 19 October 2007; accepted 13 November 2007; published online 5 December 2007)

Relation between the elastic modulus and the diameter $(D)$ of $\mathrm{ZnO}$ nanowires was elucidated using a model with the calculated $\mathrm{ZnO}$ surface stresses as input. We predict for $\mathrm{ZnO}$ nanowires due to surface stress effect: (1) when $D>20 \mathrm{~nm}$, the elastic modulus would be lower than the bulk modulus and decrease with the decreasing diameter, (2) when $20 \mathrm{~nm}>D>2 \mathrm{~nm}$, the nanowires with a longer length and a wurtzite crystal structure could be mechanically unstable, and (3) when $D<2 \mathrm{~nm}$, the elastic modulus would be higher than that of the bulk value and increase with a decrease in nanowire diameter. (C) 2007 American Institute of Physics. [DOI: 10.1063/1.2821118]

One-dimensional (1D) $\mathrm{ZnO}$ nanomaterials have significant applications as active components in nanoelectromechanical systems. ${ }^{1,2}$ The elastic modulus of $1 \mathrm{D} \mathrm{ZnO}$ nanomaterials determines how much elastic strain energy can be stored in their deformed states and further be utilized by nanodevices. However, there is no consensus about how the elastic modulus of $1 \mathrm{D} \mathrm{ZnO}$ nanomaterials should depend on their geometry and size yet. Some experimental measurements strongly indicate that the elastic modulus of $1 \mathrm{D} \mathrm{ZnO}$ nanomaterials (with sizes larger than $19 \mathrm{~nm}$ ) is lower than that of bulk $\mathrm{ZnO}^{3-7}$ In contrast, the results by Chen et al. show that the elastic modulus of $\mathrm{ZnO}$ nanowires with diameters ranging from 17 to $550 \mathrm{~nm}$ are higher than that of bulk $\mathrm{ZnO}$ and increase with the decrease of nanowire diameter. ${ }^{8}$ Atomistic modeling techniques have also been employed to study the elastic deformation in $\mathrm{ZnO}$ nanomaterials. Molecular dynamics simulations by Kulkarni et al. suggest that the elastic modulus of $\mathrm{ZnO}$ nanobelts with lateral dimensions from 1 to $4 \mathrm{~nm}$ are higher than the corresponding value of bulk $\mathrm{ZnO}$ and size-dependent. ${ }^{9}$ Zhang and Huang also found from their first-principles density functional theory (DFT) that the elastic modulus of $\mathrm{ZnO}$ nanoplates with thickness below $3 \mathrm{~nm}$ would be higher than those of bulk materials and increase as their size decreases. ${ }^{10}$

In this letter, we elucidate the size dependency of the elastic modulus of $\mathrm{ZnO}$ nanowires using the concept of surface stress. Surface stress is defined as the reversible work per unit area required to elastically stretch a surface, while surface energy is the reversible work per unit area required to create a surface. ${ }^{11}$ In Ref. 12 , the elastic modulus of a nanowire is proposed to be described in the following form:

$$
E_{\text {nanowire }}=E_{b}+\frac{8}{5} g(1-\nu) \frac{L^{2}}{D^{3}},
$$

where, $E_{\text {nanowire }}$ and $E_{b}$ are the elastic modulus of the nanowire and bulk materials, respectively, $L$ and $D$ is the length and diameter of the nanowire, $g$ is the surface stress, and $\nu$ is Poisson's ratio. Thus, a positive (tensile) surface stress would

\footnotetext{
${ }^{a)}$ Present address: Department of Mechanical Engineering, Indiana University-Purdue University Indianapolis, Indianapolis, IN 46202. Electronic mail: wang83@iupui.edu
}

lead to an increase in elastic modulus with the decreasing nanowire diameters, while a negative (compressive) surface stress would lead to a decrease in elastic modulus with the decreasing nanowire diameters. The above relation has been shown to be valid for $\mathrm{Ag}$ and $\mathrm{Pd}$ nanowires. ${ }^{12}$

Equilibrium $\mathrm{ZnO}$ has a wurtzite crystal structure. For its

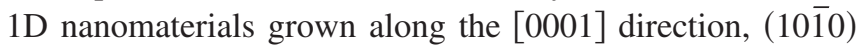
and $(11 \overline{2} 0)$ surfaces are the lateral facets. In this work, we evaluated the surface stresses for the nonpolar (1010) and $(11 \overline{2} 0)$ surfaces of $\mathrm{ZnO}$ using first-principles DFT method. The calculations were performed using the VASP code. ${ }^{13,14}$ We used the projector augmented wave method ${ }^{15}$ and the generalized gradient approximation of Perdew and Wang ${ }^{16}$ for exchange and correlation. We chose a kinetic energy cutoff of $600 \mathrm{eV}$ to expand the electronic wave functions in the plane wave basis. For (1010) surfaces, we used a supercell containing eight surface layers and eight layers of vacuum. There are two possible configurations for the (1010) surface in a wurtzite crystal: $(10 \overline{1} 0)_{A}$ (whose outermost layer is separated from its sublayer by $(\sqrt{3} / 6) a, a$ is the lattice parameter in the base plane of $\mathrm{ZnO}$ lattice) and $(10 \overline{10})_{B}$ (whose outermost layer is separated from its sublayer by $(\sqrt{3} / 3) a)$. For $(11 \overline{2} 0)$ surface, we used a supercell containing six surface layers and six layers of vacuum. For $k$-space integration, we used a $10 \times 6 \times 2 k$-point grid for (1010) surface and a $6 \times 6 \times 2 k$-point grid for $(11 \overline{2} 0)$ surface.

The structure of $\mathrm{ZnO}$ surfaces was obtained through the relaxation of atomic positions using first-principles method.

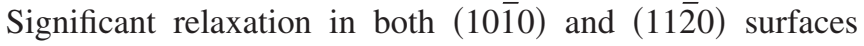
have been observed. Figures 1 and 2 show the top and side

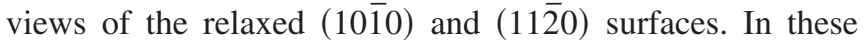
figures, big circles represent $\mathrm{Zn}$ atoms while small circles stand for $\mathrm{O}$ atoms. For illustration, the atoms in the second layer of the surfaces are drawn in gray and the atoms in the third and fourth surface layers are plotted using dashed circles.

Surface energy $(\gamma)$ and surface stress tensor $(g)$ for the relaxed $\mathrm{ZnO}$ surfaces are calculated using the approach suggested in Ref. 17 and given in Table I. The surface energy of $(10 \overline{10})_{B}$ surface is found to be much higher than the one of 
(a) Top View:
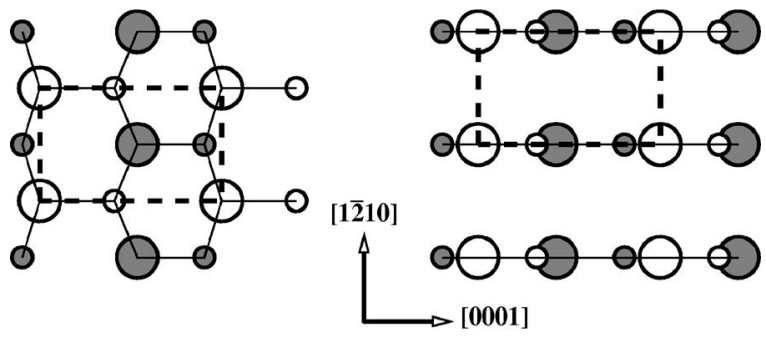

(b) Side View:
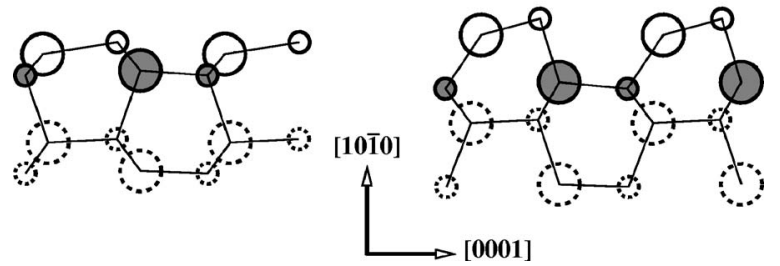

FIG. 1. (a) Top view and (b) side view of the relaxed (10̄0) surfaces of $\mathrm{ZnO}$. The left panels show the $(10 \overline{10})_{A}$ surface and the right panels show the $(10 \overline{1} 0)_{B}$ surface.

$(10 \overline{10})_{A}$ surface. This would lead $(10 \overline{10})_{A}$ to be dominant in experimental $(10 \overline{1} 0)$ surface samples and hence we assume only $(10 \overline{10})_{A}$ surfaces present in $\mathrm{ZnO}$ nanowires in this work. More importantly, the calculated surface stresses for the surfaces along the [0001] direction are negative and have the same magnitude as the surface energies. Negative surface stresses indicate that the surfaces are in compressive states and thus energetically favor expansion. Since elastic deformation increases the surface areas, those surfaces with negative surface stresses would release energy during the deformation and lower the measured elastic modulus of $1 \mathrm{D} \mathrm{ZnO}$ nanowires.

To examine the strain effect on surface energy and surface stress, we expanded $\mathrm{ZnO}(10 \overline{1} 0)_{A}$ and $(11 \overline{2} 0)$ surfaces only along the [0001] direction and relaxed the surface slabs at each given strain $(\varepsilon)$. In Fig. 3, we plot the calculated surface energies and surface stresses as a function of the strains along the [0001] direction. It is seen in Fig. 3 for the two surfaces that the surface energies decrease with the expansion strain up to about $2.5 \%$, while the surface stresses

(a) Top View:

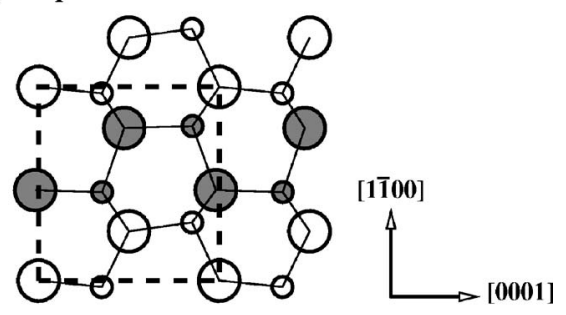

(b) Side View:

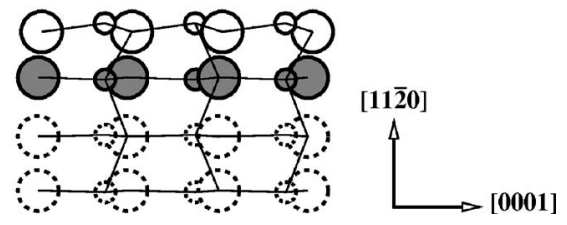

FIG. 2. (a) Top view and (b) side view of the relaxed (1120) surface of $\mathrm{ZnO}$.
TABLE I. Calculated surface energies $\gamma$ (in $\mathrm{J} / \mathrm{m}^{2}$ ) and principal surface stresses $g\left(\right.$ in $\left.\mathrm{J} / \mathrm{m}^{2}\right)$ for nonpolar (1010) and (1120) surfaces of $\mathrm{ZnO}$ using first-principles method.

\begin{tabular}{ccccc}
\hline \hline Surface & & $(10 \overline{1} 0)_{A}$ & $(10 \overline{1} 0)_{B}$ & $(11 \overline{2} 0)$ \\
\hline \multirow{2}{*}{$\gamma$} & & 0.88 & 2.35 & 0.92 \\
\multirow{2}{*}{$g$} & {$[0001]$ axis } & -1.13 & -0.91 & -0.81 \\
& {$[1 \overline{2} 10]$ axis } & -1.23 & 0.03 & $\cdots$ \\
& {$[1 \overline{1} 00]$ axis } & $\cdots$ & $\cdots$ & -0.34 \\
\hline \hline
\end{tabular}

(along the [0001] direction) increase with the strain and change signs (from negative to positive) at the strain about $1.25 \%$. Our results in Fig. 3 qualitatively point out that along the [0001] direction, the nonpolar surfaces with a strain below $1.25 \%$ would facilitate elastic deformation and soften the elastic modulus of $1 \mathrm{D} \mathrm{ZnO}$ nanomaterials. In contrast, the surfaces with a strain above $1.25 \%$ would hinder elastic deformation and harden the elastic modulus of $1 \mathrm{D} \mathrm{ZnO}$ nanomaterials.

Furthermore, we derived the size dependency of the elastic modulus for $\mathrm{ZnO}$ nanowires enclosed only by either $(10 \overline{1} 0)_{A}$ or $(11 \overline{2} 0)$ surfaces.

(a) Determination of the strain in surfaces. The energy of $\mathrm{ZnO}$ nanowires per unit length is expressed as

$$
U=U_{0}+\frac{\pi D^{2}}{4} \Delta E_{b}(\varepsilon)+\pi D \gamma(\varepsilon),
$$

where, $U_{0}$ is the energy of the equilibrium bulk $\mathrm{ZnO}$ with the same number of atoms as the nanowire, $\Delta E_{b}(\varepsilon)$ is the energy density change with [0001] strain for bulk $\mathrm{ZnO}, \gamma(\varepsilon)$ is the surface energy, and $D$ is the diameter of the nanowire. Both $\Delta E_{b}(\varepsilon)$ and $\gamma(\varepsilon)$ were calculated using first-principles method and can be accurately fitted to quadratic functions: $\Delta E_{b}(\varepsilon)$ has the minimum value at $\varepsilon=0$ while $\gamma(\varepsilon)$ [Fig. 3(a)] reach the minimum values at $\varepsilon$ about $2.5 \%$. Therefore, we could determine the strain $\left(\varepsilon^{*}\right)$ in the $\mathrm{ZnO}$ nanowire surfaces through $(\partial U / \partial \varepsilon)\left(\varepsilon^{*}\right)=0$.

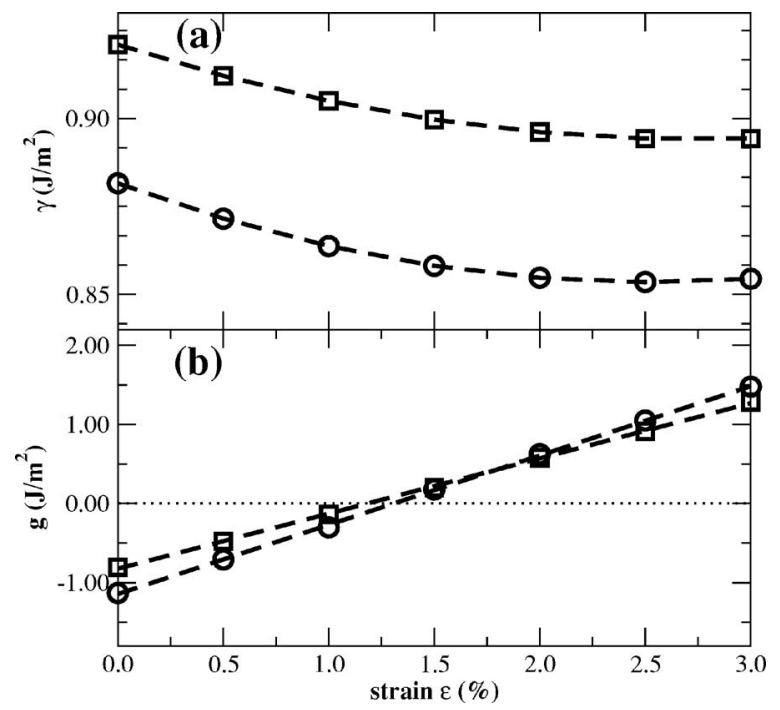

FIG. 3. Variation of (a) surface energies $(\gamma)$ and (b) surface stresses $(g)$ in [0001] axis of $\mathrm{ZnO}$ surfaces with the strains along the [0001] direction. Circles represent the data for $(10 \overline{1} 0)_{A}$ surface and squares represent the data for $(11 \overline{2} 0)$ surface. In panel (a), dashed lines are the fitting curves using a quadratic function. In panel (b), dashed lines are the linear fitting of the data. 


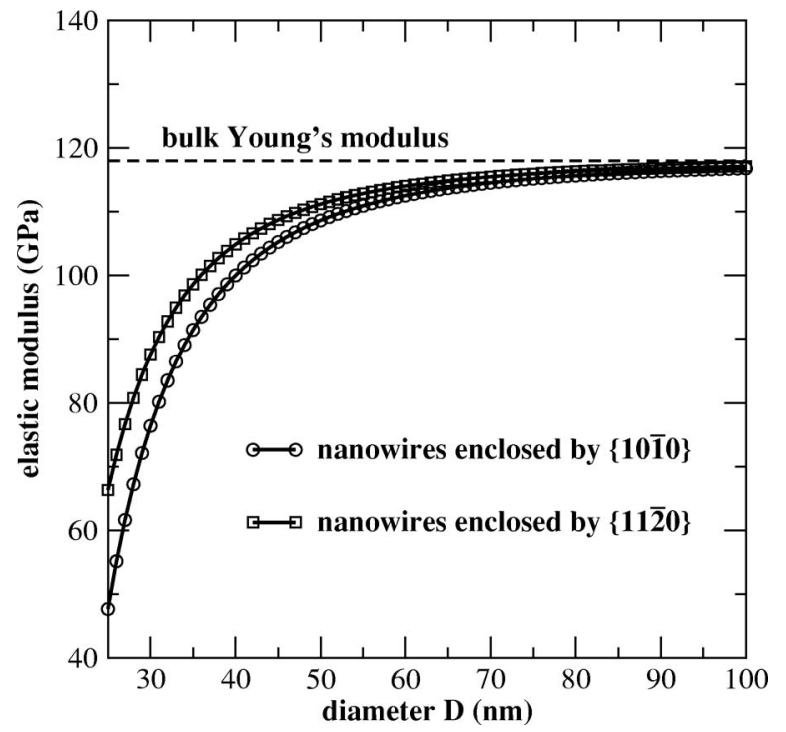

FIG. 4. Size dependency of the elastic modulus of $\mathrm{ZnO}$ nanowires enclosed by $(10 \overline{10})_{A}$ surfaces (circles) or $(11 \overline{2} 0)$ surfaces (squares).

(b) Determination of surface stress. Figure 3(b) shows that the surface stresses of $\mathrm{ZnO}$ are linearly dependent on the strain $\varepsilon^{*}$ in the surfaces.

(c) Calculation of the elastic modulus of $\mathrm{ZnO}$ nanowires using Eq. (1). In this work, we assume $L=1000 \mathrm{~nm}$, which is the typical suspended length of $1 \mathrm{D} \mathrm{ZnO}$ nanomaterials in atomic force microscopy three-point bending tests. ${ }^{6}$ We also assume $E_{b}=118 \mathrm{GPa}$ and $\nu=0.32$ calculated using the experimental elastic constants. ${ }^{18}$

In Fig. 4, we plot the calculated elastic modulus of $\mathrm{ZnO}$ nanowires as a function of their diameters. It is predicted that due to surface stress effect, the elastic modulus of $\mathrm{ZnO}$ nanowires $(D>25 \mathrm{~nm})$ would be lower than the Young's modulus of bulk $\mathrm{ZnO}$ and decreases with the decreasing diameters. Our theoretical predictions are consistent with most of the early experimental measurements. ${ }^{3-7}$ Especially, our predictions agree excellently with the recent systematic measurements on the elastic properties of $\mathrm{ZnO}$ nanobelts. ${ }^{19}$ Figure 3(e) in that work shows that the elastic modulus of $\mathrm{ZnO}$ nanobelts is lower than the bulk value and will decrease with a decrease in their thickness (equivalent to nanowire diameter).

Our model was derived based on linear elasticity theory and could not accurately make quantitative predictions for small-diameter $(D<25 \mathrm{~nm})$ nanowires, in which nonlinear relaxation effect would be pronounced. Nevertheless, we can qualitatively discuss two issues using our model. (1) Our model gives negative elastic modulus for the $\mathrm{ZnO}$ nanowires with diameters below $20 \mathrm{~nm}$ but larger than $2 \mathrm{~nm}$, implying those nanowires (with a wurtzite crystal structure and a length longer than $1000 \mathrm{~nm}$ ) mechanically unstable. It seems to be consistent with the previous findings that $\mathrm{ZnO}$ nanomaterials would undergo a phase transformation from wurtzite to graphite crystal structures below some critical size. ${ }^{20,21}$ (2) For $\mathrm{ZnO}$ nanowires with a diameter below $2 \mathrm{~nm}$, the predicted strains in their surfaces are larger than $1.25 \%$ and hence the surface stress will be positive. Our model predicts that the elastic modulus of those $\mathrm{ZnO}$ nanowires will be higher than the bulk value and increase with the decreasing nanowire diameters, agreeing well with the previous theoretical calculations ${ }^{9,10}$
As a further note, we anticipate that surface stress would play a similar role in determining the elastic modulus of some other wurtzite crystal 1D nanomaterials. For instance, it has also been experimentally found that $1 \mathrm{D} \mathrm{ZnS} \mathrm{(Refs.} 22$ and 23) and GaN (Refs. 24-26) nanomaterials exhibit lower elastic modulus compared to their bulk values.

In conclusion, combining first-principles calculations and linear elasticity theory, we revealed that the surface enclosed large-diameter $\mathrm{ZnO}$ nanowires would release energy when stretched, and that is the reason for the measured low elastic modulus of $1 \mathrm{D} \mathrm{ZnO}$ nanomaterials. Moreover, we pointed out that the surface stress would be positive and hence predicted enhanced elastic modulus for the smalldiameter $\mathrm{ZnO}$ nanowires. In the context of surface stress, we resolved the "apparent" contradictions on the elastic property of $\mathrm{ZnO}$ nanomaterials between previous experimental measurements ${ }^{3-7}$ (which found low elastic modulus for large-size nanomaterials) and atomistic simulations 9,10 (which predicted enhanced elastic modulus for small-size nanomaterials). Therefore, surface stress plays a very important role in determining the elastic modulus of $1 \mathrm{D} \mathrm{ZnO}$ nanomaterials, suggesting that the elastic properties of nanomaterials could be engineered by altering the surface stress through rational control of the adsorptions, charges, structure, and impurities in the surfaces.

G. Wang thanks the University of South Carolina Aiken for financial support. X.D. Li acknowledges the research grants from the National Science Foundation (Grant Nos. EPS-0296165 and 0653651), the ACS Petroleum Research Fund (ACS PRF\#40450-AC10), and the University of South Carolina NanoCenter.

${ }^{1}$ Z. L. Wang and J. Song, Science 312, 242 (2006).

${ }^{2}$ X. Wang, J. Song, J. Liu, and Z. L. Wang, Science 316, 102 (2007).

${ }^{3}$ X. D. Bai, P. X. Gao, Z. L. Wang, and E. G. Wang, Appl. Phys. Lett. 82, 4806 (2003).

${ }^{4}$ J. Song, X. Wang, E. Riedo, and Z. L. Wang, Nano Lett. 5, 1954 (2005).

${ }^{5}$ M. H. Zhao, C. B. Jiang, S. X. Li, and S. X. Mao, Mater. Sci. Eng., A 409, 223 (2005).

${ }^{6} \mathrm{H}$. Ni and X. D. Li, Nanotechnology 17, 3591 (2006).

${ }^{7}$ W. Mai and Z. L. Wang, Appl. Phys. Lett. 89, 073112 (2006).

${ }^{8}$ C. Q. Chen, Y. Shi, Y. S. Zhang, J. Zhu, and Y. J. Yan, Phys. Rev. Lett. 96, 075505 (2006).

${ }^{9}$ A. J. Kulkarni, M. Zhou, and F. J. Ke, Nanotechnology 16, 2749 (2005).

${ }^{10}$ L. Zhang and H. C. Huang, Appl. Phys. Lett. 89, 183111 (2006).

${ }^{11}$ R. C. Cammarata, Prog. Surf. Sci. 1, 1 (1994).

${ }^{12}$ S. Cuenot, C. Frétigny, S. Demoustier-Champagne, and B. Nysten, Phys. Rev. B 69, 165410 (2004).

${ }^{13}$ G. Kresse and J. Hafner, Phys. Rev. B 47, 558 (1993).

${ }^{14}$ G. Kresse and J. Furthmüller, Phys. Rev. B 54, 11169 (1996).

${ }^{15}$ G. Kresse and D. Joubert, Phys. Rev. B 59, 1758 (1999).

${ }^{16}$ J. P. Perdew and Y. Wang, Phys. Rev. B 45, 13244 (1992).

${ }^{17}$ R. J. Needs, Phys. Rev. Lett. 58, 53 (1987).

${ }^{18}$ T. B. Bateman, J. Appl. Phys. 33, 3309 (1962).

${ }^{19}$ M. Lucas, W. Mai, R. Yang, Z. L. Wang, and E. Reido, Nano Lett. 7, 1314 (2007).

${ }^{20}$ A. J. Kulkarni, M. Zhou, K. Sarasamak, and S. Limpijumnong, Phys. Rev. Lett. 97, 105502 (2006).

${ }^{21}$ L. Zhang and H. C. Huang, Appl. Phys. Lett. 90, 023115 (2007).

${ }^{22}$ X. D. Li, X. Wang, Q. Xiong, and P. C. Eklund, Nano Lett. 5, 1982 (2005).

${ }^{23}$ Q. Xiong, N. Duarte, S. Tadigadapa, and P. C. Eklund, Nano Lett. 5, 1904 (2006).

${ }^{24}$ C. Y. Nam, P. Jaroenapibal, D. Tham, D. E. Luzzi, S. Evoy, and J. E. Fischer, Nano Lett. 6, 153 (2006).

${ }^{25}$ H. Ni, X. D. Li, G. S. Cheng, and R. Klie, J. Mater. Res. 21, 2882 (2006).

${ }^{26}$ G. Feng, W. D. Nix, Y. Yoon, and C. J. Lee, J. Appl. Phys. 99, 074304 (2006). 